\title{
MYC Box
}

National Cancer Institute

\section{Source}

National Cancer Institute. MYC Box. NCI Thesaurus. Code C13406.

MYC boxes I and II, found in the N termini of all members of the MYC protein family, function to direct the degradation of the c-MYC protein. Both MYC boxes I and II contain sufficient information to independently direct the degradation of otherwise stably expressed proteins to which they are fused. At least part of the MYC box-directed degradation occurs via the proteasome. 